\title{
On the Modal and Non-Modal Model Reduction of Metallic Structures with Variable Boundary Conditions
}

\author{
Wolfgang Witteveen \\ University of Applied Sciences Upper Austria, Wels, Austria \\ Email: wolfgang.witteveen@fh-wels.at
}

Received October 1, 2012; revised November 2, 2012; accepted November 12, 2012

\begin{abstract}
Vibration mode based model reduction methods like component mode synthesis (CMS) will be compared to methods coming from control engineering, namely moment matching (MM) and balanced truncation (BT). Conclusions based on the theory together with a numerical demonstration will be presented. The key issues on which the paper is focused are the reduction of metallic structures, the sensitivity of the reduced model to varying boundary conditions, full system response, accurate statics and the possibility to determine "a priori" the number of needed modes (trial vectors). These are important topics for the use of reduction methods in general and in particular for the implementation of FE models in multi body system dynamics where model reduction is widely used. The intention of this paper is to give insight into the methods nature and to clarify the strengths and limitations of the three methods. It turns out, that in the considered framework CMS delivers the best results together with a clear strategy for an "a priori" selection of the modes (trial vectors).
\end{abstract}

Keywords: Model Reduction; Vibration Modes; Component Mode Synthesis; Moment Matching; Balanced Truncation

\section{Introduction}

Model order reduction is a key issue when the dynamic of finite element (FE) models is investigated. It is concerned with the question whether a system with a large number of degrees of freedom (DOF) $n$ can be accurately represented by a transformed system of the size $m$ with $m$ $<<n$, see [1-3] for a general review and introduction on this topic. During the last decades a lot of reduction methods have been proposed. For linear elastic structures with a moderate number of input degrees of freedom (DOF), mode based reduction methods, like Component mode synthesis (CMS) have been developed to very reliable standard tools. An overview of the family of CMS methods is given by Craig in $[4,5]$. Another, more recently published review has been done by de Clerk [6]. All CMS variants have in common, that at least a part of the reduction base is formed by vibration modes. Therefore this family of reduction methods will be denoted as "mode" based within this work.

In the last few years a lot of effort has been made in order to adapt model reduction methods for mechanical structures which come originally from control engineering, namely moment matching (MM) and balanced truncation (BT). In contrast to CMS where the trial vectors have a physical meaning, the latter two methods are based on mathematical considerations. Therefore MM and BT are denoted as "non-modal" reduction methods. In the following both approaches will be outlined as far as it is necessary to understand the principal characteristics and their consequences on the topic of this work. More detailed introductions into MM which is also known as "Krylov subspace method" can be found in [7-11] and in case of BT the reader is referred to [12-15] for more background information. In some newer publications [16-19] the three methods have been compared to each other and MM and BT have been pointed out as kind of the "better method" with less error. In case of BT it has been emphasized, that a "a priori" error bound in term of displacements can be given, see among others [10,20,21].

This work deals with a critical qualitative comparison of the three methods for industrial use of model reduction for metallic structures with focus on the following important issues:

1) Sensitivity of the reduced system with respect to varying boundary conditions;

2) Full system response;

3) Static and dynamic response;

4) Simple and clear strategy for an "a priori" selection of the number of needed trial vectors.

1) Sensitivity of the reduced system with respect to varying boundary condition: This is of particular importance for multi body dynamics where the implementation of the component in the overall system is in general not 
known at the time when the reduction base is computed. In this particular case an arbitrary interaction of the input/output DOF with the surrounding rigid or flexible bodies should be possible. The input/output DOF are those on which external forces may be applied on the structure. In other words, the response of the reduced system has to be sufficient accurate independent of the stiffness or mass which may be applied to the input/output DOF.

2) Full system response: MM and BT guarantee a defined behavior between the input and output (I/O) of the reduced system. The output vector holds a linear superposition of the state vector and represents the quantity of interest. This is of special interest in control engineering but not sufficient in structural mechanics where accurate stress recovery is an important requirement. For details on stress recovery of reduced components, see [22-24]. Consequently, the response of the entire structure needs to be considered because the place of the highest stress is not known a priory and is not necessarily represented by the $\mathrm{I} / \mathrm{O}$. For this reason this work is not focused on the $\mathrm{I} / \mathrm{O}$ behavior but on the full system response.

3) Static and dynamic response: In mechanics the static response of a system is of special interest. It is not just a theoretical issue as it may be in other disciplines. A static portion in the overall system response leads to a mean stress which influences the overall stress and therefore the components lifetime. Neglecting the static in general is not an option from a mechanical engineers point of view.

4) Simple and clear strategy for an "a priori" selection of the number of needed trial vectors: A key feature of a reliable reduction method is a simple way to determine the number of required modes in order to get an accurate solution. No application engineer is interested in doing a convergence analysis or a comparison with the full system, as it is common in the academic society.

Note, that the applicability of the methods for huge FE models is not issue of the current work, even it is still a field of research, see [14] in case of BT. The focus is on basic insights and qualitative differences.

There is already some comparative literature available, see for example [25] and, as mentioned [16-19]. To the best knowledge of the author the following points of this paper have not been investigated and pointed out yet:

1) A comparison of the three methods with the focus on the issues mentioned above, especially the varying boundary conditions which are of significant importance for the use of model reduction in multi body simulation.

2) The reliability of MM and BT in terms of statics.

3) The practical significance of the error bound of BT when the boundary conditions of the input DOF in the reduced system divers significant from the situation at the time of trial vector generation.
4) The reliability of the methods when large parts of a structure are not represented by the I/O. This is of particular interest when large models like car bodies or aircrafts with few I/O locations will be reduced.

A preliminary und unfinished version of this work has been published at the SEM IMAC Conference in Jacksonville, FL, US [26].

The paper is organized as follows: In a first section general issues connected to model reduction will be discussed as well as issues arising from the prior mentioned topics. In the subsequent section all three approaches will be briefly outlined as far as it is necessary to see the qualitative differences. At the end of this chapter the most important conclusions already can be drawn based on the given equations. In the following a simple beam example is used to demonstrate the expected advantages and disadvantages of each method which leads to a clear conclusion and recommendation. Finally some publications will be briefly discussed.

\section{General Issues on Model Reduction of Metallic Structures}

The FE method leads to an equation of motion in the form of

$$
\begin{aligned}
& M \ddot{x}+K \boldsymbol{x}=B_{1} u \\
& y=C_{1} x
\end{aligned}
$$

where the $(n \times n)$ matrixes $\boldsymbol{M}$ and $\boldsymbol{K}$ are the mass and stiffness matrix, the $(n \times 1)$ vector $\boldsymbol{x}$ contains the bodies DOF, the $(u \times 1)$ vector $\boldsymbol{u}$ holds the time history of the applied loads, the $(n \times u)$ matrix $\boldsymbol{B}_{1}$ maps the loads to the corresponding degrees of freedom, the $(y \times 1)$ vector $\boldsymbol{y}$ holds the output of interest and $\boldsymbol{C}_{1}$ maps the state variables to the output. In the following a symmetric system is considered with $\boldsymbol{M}=\boldsymbol{M}^{T}$ and $\boldsymbol{K}=\boldsymbol{K}^{T}$. In next subsection it will be discussed why no viscous damping is considered for the reduction process.

The corresponding reduced system can be given as

$$
\begin{aligned}
& \tilde{\boldsymbol{M}} \dot{\boldsymbol{z}}+\tilde{\boldsymbol{K}}_{\boldsymbol{z}}=\tilde{\boldsymbol{B}}_{1} \boldsymbol{u} \\
& \boldsymbol{y}=\tilde{\boldsymbol{C}}_{1} \boldsymbol{z} \\
& \boldsymbol{x}=\boldsymbol{V} \boldsymbol{z}, \quad \tilde{\boldsymbol{M}}=\boldsymbol{W}^{T} \boldsymbol{M} \boldsymbol{V}, \quad \tilde{\boldsymbol{K}}=\boldsymbol{W}^{T} \boldsymbol{K} \boldsymbol{V}, \\
& \tilde{\boldsymbol{B}}_{1}=\boldsymbol{W}^{T} \boldsymbol{B}_{1}, \quad \tilde{\boldsymbol{C}}_{1}=\boldsymbol{C}_{1} \boldsymbol{V}
\end{aligned}
$$

with the $(m \times 1)$ vector $z$ and the $(n \times m)$ matrixes $\boldsymbol{V}$ and $\boldsymbol{W}$. Model order reduction deals with the question of determining the matrixes $\boldsymbol{V}$ and $\boldsymbol{W}$, so that the reduced system captures somehow the important characteristics of the unreduced system.

For a better readability of this work it is remembered that (1) can be transformed into a first order system in the form of 


$$
\begin{aligned}
& \overline{\boldsymbol{E}} \dot{\boldsymbol{q}}=\overline{\boldsymbol{A}} \boldsymbol{q}+\boldsymbol{B u} \\
& \boldsymbol{y}=\boldsymbol{C} \boldsymbol{q} \\
& \boldsymbol{q}^{T}=\left[\begin{array}{ll}
\boldsymbol{x}^{T} & \dot{\boldsymbol{x}}^{T}
\end{array}\right], \boldsymbol{B}=\left[\begin{array}{c}
\mathbf{0} \\
\boldsymbol{B}_{1}
\end{array}\right], \boldsymbol{C}=\left[\begin{array}{ll}
\boldsymbol{C}_{1} & \mathbf{0}
\end{array}\right] \\
& \overline{\boldsymbol{E}}=\left[\begin{array}{cc}
\boldsymbol{I} & \mathbf{0} \\
\mathbf{0} & \boldsymbol{M}
\end{array}\right], \overline{\boldsymbol{A}}=\left[\begin{array}{cc}
\mathbf{0} & \boldsymbol{I} \\
-\boldsymbol{K} & \mathbf{0}
\end{array}\right]
\end{aligned}
$$

In [15] a little modification of (3) is suggested, so that symmetric system matrixes $\boldsymbol{E}$ and $\boldsymbol{A}$ are obtained:

$$
\begin{aligned}
& E \dot{q}=A q+B u \\
& \boldsymbol{y}=\boldsymbol{C q} \\
& E=\left[\begin{array}{cc}
-\boldsymbol{K} & 0 \\
0 & M
\end{array}\right], \quad \boldsymbol{A}=\left[\begin{array}{cc}
0 & -\boldsymbol{K} \\
-\boldsymbol{K} & 0
\end{array}\right]
\end{aligned}
$$

The latter modification has some important implications which will be reported in subsection 2.2. The according reduced system is

$$
\begin{aligned}
& \tilde{\boldsymbol{E}} \dot{\tilde{\boldsymbol{q}}}=\tilde{\boldsymbol{A}} \tilde{\boldsymbol{q}}+\tilde{\boldsymbol{B}} \boldsymbol{u} \\
& \boldsymbol{y}=\tilde{\boldsymbol{C}} \tilde{\boldsymbol{q}} \\
& \tilde{\boldsymbol{q}}=\boldsymbol{V} \boldsymbol{q}, \tilde{\boldsymbol{E}}=\boldsymbol{W}^{T} \boldsymbol{E} \boldsymbol{V}, \tilde{\boldsymbol{A}}=\boldsymbol{W}^{T} \boldsymbol{A} \boldsymbol{V} \\
& \tilde{\boldsymbol{B}}=\boldsymbol{W}^{T} \boldsymbol{B}, \tilde{\boldsymbol{C}}=\boldsymbol{C} \boldsymbol{V}
\end{aligned}
$$

with the $(2 n \times m)$ reduction matrixes $\boldsymbol{V}$ and $\boldsymbol{W}$.

\subsection{Absence of the Viscose Damping Matrix}

The damping matrix is not considered in Equation (1) because of the nature of the two most frequently involved dissipation mechanisms, namely material damping and joint damping (micro slip). Both of them are energy dissipation mechanisms which cannot be properly modeled by pure viscous damping. Material damping of metallic structures will be overestimated in case of high frequency if it is modeled with a viscous damping matrix, see [27-29] for an experimental verification with aluminum. The reason why material damping is commonly modeled with a velocity proportional approach is more its simplicity than its accuracy. Furthermore it is mention worth that material damping of metallic structures is that low so that it is normally dominated by other damping mechanisms like joint damping or by external applied damping like rubber bearings, lubrication bearings or the like. Joint damping (micro slip) is frequently independent and cannot be modeled by a viscous damping at all, see exemplarily [30]. Consequently, a viscous damping is always an inaccurate approximation for metallic structures and it does not matter if it is introduced in the full system or in the reduced system. When the damping matrix is regarded in the reduction process, it will influence the final comparison between the reduced and the full model. This doesn't make sense because something physi- cally not meaningful is introduced and may affect an objective evaluation. Note, that the latter considerations may not be valid for structures with a large amount of rubber, plastic or other visco-elastic materials.

\subsection{Stability and Symmetry of the Reduced System}

If the reduced model (2) is used for time integration it is of significant importance, that no instability will be introduced due to the model reduction. In this particular case, when no damping is regarded the eigenvalues of the reduced model are required to be real, such as they are in the original system. The symmetry of a mechanical system is a fundamental characteristic which should be preserved during the reduction process.

Both is obtained in case of a so called "symmetric or single sided projection" which requires an identical reduction and test space matrix

$$
\boldsymbol{W}=\boldsymbol{V}
$$

In case of a symmetric system (4) and when the input DOF are the same as the output DOF $\left(\boldsymbol{C}_{1}^{T}=\boldsymbol{B}_{1}\right)$ it has been demonstrated in [7] and [15], that $\mathrm{MM}$ and BT leads to a symmetric projection (6).

Note, that the latter, more mathematical considerations do have a mechanical pendant. In case of a coordinate transformation it is common in mechanics to apply the principle of virtual work, which requires, that the work of all forces due to a virtual displacement has to vanish. This can be written as

$$
\delta \boldsymbol{x}^{T}\left(\boldsymbol{M} \ddot{\boldsymbol{x}}+\boldsymbol{K} \boldsymbol{x}-\boldsymbol{B}_{1} \boldsymbol{u}\right)=\mathbf{0}
$$

where $\delta$ indicates a virtual displacement of the state vector. If the reduction law $\boldsymbol{x}=\boldsymbol{V} \boldsymbol{z}$ is used for the state vector as well as for the virtual displacement the reduced system (2) is obtained with $\boldsymbol{W}=\boldsymbol{V}$. For the latter reasons we restrict ourselves to such systems where $\boldsymbol{C}_{1}^{T}=\boldsymbol{B}_{1}$.

\section{Brief Review on Component Mode Synthesis, Moment Matching and Balanced Truncation}

For the sake of simplicity and readability an invertible stiffness and mass matrix will be assumed further one. The presence of rigid body modes doesn't have an effect on the conclusions which will be drawn. As mentioned before, the theory given next is by far not exhaustive but should give at least as much insight, that the finally drawn conclusions are understandable.

\subsection{Component Mode Synthesis, See [4-6]}

A CMS based reduction is typically directly applied to the second order system (1). Commonly, the reduction matrix is of the form 


$$
\boldsymbol{V}=\left[\begin{array}{ll}
\boldsymbol{V}_{S} & \boldsymbol{V}_{D}
\end{array}\right]
$$

where the trial vectors in the $(\mathrm{n} \times \mathrm{p})$ matrix $\boldsymbol{V}_{D}$ are vibration modes which capture the systems dynamics. These modes are obtained by a proper eigenvalue problem which delivers the modes eigenfrequencies. For the reduction process just those $p$ modes $(p<<n)$ are regarded, having an eigenfrequency smaller as a defined limit, which typically a factor of $1.5-2$ higher as the highest relevant frequency content of all involved forces.

In order to improve the convergence, some static trial vectors are added to the vibration modes. These trial vectors are obtained by loads on the input/output DOF and collected in the columns of the $(n \times s)$ matrix $\boldsymbol{V}_{S}$.

During the last decades a lot of methods for the computation of $\boldsymbol{V}$ according to (8) have been suggested. An overview is given by Craig in [5]. In the latter publication it has been shown, that the different approaches span a similar space. For this publication the most frequently used method has been chosen to represent the family of "mode based" reduction methods, namely the fixed boundary CMS introduced by Craig (and Bampton) [31].

According to Craig's method the vector of nodal DOF of the FE model is subdivided into

$$
\boldsymbol{x}^{T}=\left[\begin{array}{ll}
\boldsymbol{x}_{B}^{T} & \boldsymbol{x}_{I}^{T}
\end{array}\right]
$$

where the $(b \times 1)$ vector $\boldsymbol{x}_{B}$ represents the "boundary" DOF and the $((n-b) \times 1)$ vector $\boldsymbol{x}_{I}$ holds the remaining, inner DOF. The boundary DOF is those DOF on which external forces may be applied. In this work these DOF are mostly denoted as input/output DOF. In terms of (1), the input matrix $\boldsymbol{B}_{1}$ contains non zero entries at these DOF. According to the subdivision (9), an eigenvalue problem in the form of

$$
\left[\boldsymbol{K}_{I, I}-\omega_{i}^{2} \boldsymbol{M}_{I, I}\right] \boldsymbol{v}_{D, i}=\mathbf{0}
$$

can be used for the computation of the vibration modes. The $((n-b) \times(n-b))$ matrixes $\boldsymbol{K}_{I, I}$ and $\boldsymbol{M}_{I, I}$ denote the portion of $\boldsymbol{K}$ and $\boldsymbol{M}$ matching the definition of (9). The obtained modes $\boldsymbol{v}_{D, i}$ are named as ,Fixed Boundary Normal Modes' and can be given as

$$
\boldsymbol{V}_{D}=\left[\begin{array}{cccc}
\mathbf{0} & \mathbf{0} & \mathbf{0} & \mathbf{0} \\
\boldsymbol{v}_{D, 1} & \boldsymbol{v}_{D, 2} & \cdots & \boldsymbol{v}_{D, p}
\end{array}\right]
$$

with an user defined eigenfrequency limit $\omega^{*}$ so that

$$
\omega_{p}<\omega^{*}
$$

As mentioned before, the latter limit is usually connected to the highest relevant frequency content of the excitation. Die Matrix $\boldsymbol{V}_{S}$ is obtained according to a Guyan [32] reduction

$$
\boldsymbol{V}_{S}=\left[\begin{array}{c}
\boldsymbol{I} \\
-\boldsymbol{K}_{I, I}^{-1} \boldsymbol{K}_{I, B}
\end{array}\right]
$$

with the $(b \times b)$ identity matrix $I$. Obviously, each boundary DOF introduces a trial vector in $\boldsymbol{V}_{S}$.

It is known that the use of such static displacement fields significantly improve the convergence of the solution in case of imposed boundary conditions which are different to the one at the time of mode generation, see reference [24]. Therefore it is to expect, that CMS should deliver good results independently of the applied boundary conditions. This is an important feature for accurate stress recovery as well, see [24].

Unfortunately no "a priori" error bound in terms of displacement is known yet. However, the procedure ensures exact statics and the presence of all modes of interest within a well defined frequency range.

\subsection{Moment Matching, See [7-11]}

With the Laplace transformation the transfer function of the system (4) can be given as

$$
\boldsymbol{H}(s)=\boldsymbol{C}(s \boldsymbol{E}-\boldsymbol{A})^{-1} \boldsymbol{B}
$$

where $\mathrm{s}$ holds the complex Laplace variable. An approximation of (14) with a Power Series around $s_{0}$ gives

$$
\boldsymbol{H}(s)=\sum_{j=0}^{\infty} \boldsymbol{T}_{j}^{s_{0}}\left(s_{0}-s\right)^{j}
$$

with the so called $j$-th moment.

$$
\boldsymbol{T}_{j}^{s_{0}}=\boldsymbol{C}\left(\left(s_{0} \boldsymbol{E}-\boldsymbol{A}\right)^{-1} \boldsymbol{E}\right)^{j}\left(s_{0} \boldsymbol{E}-\boldsymbol{A}\right)^{-1} \boldsymbol{B}
$$

see [7]. If $\boldsymbol{W}$ and $\boldsymbol{V}$ are chosen in such a way that the spanned space is equal to

$$
\begin{aligned}
\boldsymbol{V} & =\left[\begin{array}{llll}
\boldsymbol{v}_{m-1} & \cdots & \boldsymbol{v}_{1} & \boldsymbol{v}_{0}
\end{array}\right] \\
& =\left[\begin{array}{llll}
\boldsymbol{P}_{v}{ }^{m-1} \boldsymbol{R}_{v} & \cdots & \boldsymbol{P}_{v}^{1} \boldsymbol{R}_{v} & \boldsymbol{R}_{v}
\end{array}\right] \\
\boldsymbol{W} & =\left[\begin{array}{llll}
\boldsymbol{w}{ }_{m-1} & \cdots & \boldsymbol{w}_{1} & \boldsymbol{w}_{0}
\end{array}\right] \\
& =\left[\begin{array}{lllll}
\boldsymbol{P}_{W}{ }^{m-1} \boldsymbol{R}_{W} & \cdots & \boldsymbol{P}_{W}{ }^{1} \boldsymbol{R}_{W} & \boldsymbol{R}_{W}
\end{array}\right]
\end{aligned}
$$

with

$$
\begin{aligned}
& \boldsymbol{R}_{v}=\left(s_{0} \boldsymbol{E}-\boldsymbol{A}\right)^{-1} \boldsymbol{B} \\
& \boldsymbol{P}_{v}=\left(s_{0} \boldsymbol{E}-\boldsymbol{A}\right)^{-1} \boldsymbol{E} \\
& \boldsymbol{R}_{W}=\left(s_{0} \boldsymbol{E}-\boldsymbol{A}\right)^{-T} \boldsymbol{C}^{T} \\
& \boldsymbol{P}_{v}=\left(s_{0} \boldsymbol{E}-\boldsymbol{A}\right)^{-T} \boldsymbol{E}^{T}
\end{aligned}
$$

it can be shown, that the first $2 \mathrm{~m}$ moments of the reduced system around $s_{0}$ are equal to those in the full system.

$$
\boldsymbol{T}_{j}^{s_{0}}=\tilde{\boldsymbol{T}}_{j}^{s_{0}} \quad j=1, \cdots, 2 m
$$

Note, that a direct implementation of (17) for the construction of $\boldsymbol{W}$ and $\boldsymbol{V}$ is numerically disadvantageous. The literature offers better choices, see exemplarily [11]. 
The trial vectors of (17) are also called "Krylov sequences". When $\boldsymbol{M}=\boldsymbol{M}^{T}, \boldsymbol{K}=\boldsymbol{K}^{T}$ and $\boldsymbol{C}^{T}=\boldsymbol{B}$ it easily can be seen, that $\boldsymbol{W}$ evaluates exactly to $\boldsymbol{V}$, see (17) and (18).

The transfer function of the second order system (1) is

$$
\boldsymbol{H}(s)=\boldsymbol{C}_{1}\left(s^{2} \boldsymbol{M}-(-\boldsymbol{K})\right)^{-1} \boldsymbol{B}_{1}
$$

The formal similarity of (20) and (14) can be used to obtain the desired quantities in a similar matter. Again $\boldsymbol{W}$ evaluates to $\boldsymbol{V}$ in case of a symmetric system $\left(\boldsymbol{M}=\boldsymbol{M}^{T}, \boldsymbol{K}\right.$ $=\boldsymbol{K}^{T}$ ) and when the input is equal to the output $\left(\boldsymbol{C}_{1}^{T}=\boldsymbol{B}_{1}\right)$.

$$
\begin{aligned}
\boldsymbol{V} & =\left[\begin{array}{llll}
\boldsymbol{v}_{m-1} & \cdots & \boldsymbol{v}_{1} & \boldsymbol{v}_{0}
\end{array}\right] \\
& =\left[\begin{array}{llll}
\boldsymbol{P}^{m-1} \boldsymbol{R} & \cdots & \boldsymbol{P}^{1} \boldsymbol{R} & \boldsymbol{R}
\end{array}\right] \\
\boldsymbol{R} & =\left(s_{0}^{2} \boldsymbol{M}+\boldsymbol{K}\right)^{-1} \boldsymbol{B}_{1} \\
\boldsymbol{P} & =\left(s_{0}^{2} \boldsymbol{M}+\boldsymbol{K}\right)^{-1} \boldsymbol{M}
\end{aligned}
$$

The computation of $\boldsymbol{V}$ in the presence of a damping matrix can be found in [7].

A more mechanical interpretation of the Krylov sequences around $s_{0}=0$ will be given next and can be found in [33,34]. There, each additional Krylov sequence regards the dynamic residua of the already existing Krylov subspace in a quasi-static matter. In a first step the state vector of (1) is approximated by the static only. This leads to

$$
\boldsymbol{x}=\boldsymbol{K}^{-1} \boldsymbol{B}_{1} \boldsymbol{u}+\boldsymbol{x}^{(1)}=\boldsymbol{v}_{0} \boldsymbol{u}+\boldsymbol{x}^{(1)}
$$

where $\boldsymbol{x}^{(1)}$ is the (dynamic) residuum of this quasi static approximation and $\boldsymbol{K}^{-1} \boldsymbol{B}_{1}$ is selected as first trial vector $\boldsymbol{v}_{0}$ which is scaled by $\boldsymbol{u}$. In a next step the acceleration vector of (1) is replaced by the second derivative of (22) with respect to the time. This gives

$$
\boldsymbol{M} \ddot{\boldsymbol{x}}^{(1)}+\boldsymbol{K} \boldsymbol{x}^{(1)}=\boldsymbol{M} \boldsymbol{K}^{-1} \boldsymbol{B}_{1} \ddot{\boldsymbol{u}}
$$

As before $\boldsymbol{x}^{(1)}$ is approximated by the static response in the form

$$
\boldsymbol{x}^{(1)}=\boldsymbol{K}^{-1} \boldsymbol{M} \boldsymbol{K}^{-1} \boldsymbol{B}_{1} \ddot{\boldsymbol{u}}+\boldsymbol{x}^{(2)}=\boldsymbol{v}_{1} \ddot{\boldsymbol{u}}+\boldsymbol{x}^{(2)}
$$

where $\boldsymbol{x}^{(2)}$ is the remaining (dynamic) residuum and the second trial vector $\boldsymbol{v}_{1}$ is introduced. Substituting (24) into (22) delivers

$$
\boldsymbol{x}=\boldsymbol{v}_{0} \boldsymbol{u}+\boldsymbol{v}_{1} \ddot{\boldsymbol{u}}+\boldsymbol{x}^{(2)}
$$

and it is obvious that the latter procedure can be repeated as long as the result has the desired accuracy. The obtained vectors $\boldsymbol{v}_{0}, \boldsymbol{v}_{1}, \cdots$ can be collected in a matrix $\boldsymbol{V}$. They are identical with the one of (21) in case of $s_{0}=0$.

A characteristic of a reduction base formed of Krylov sequences is the presence of the structures static response $\boldsymbol{v}_{0}$. With the same considerations as before it can be expected, which varying boundary conditions may not lead to unacceptable results. Again there is no "a priori" error bound available and furthermore no "a priori" frequency limit for the validity of the reduction base can be given. This is a drawback when it comes to the question how many trial vectors have to be selected "a priori" in order to get accurate results.

\subsection{Balanced Truncation, See $[9,10,12-15]$}

For the introduction of the Gramian matrixes the system (3) is considered with $\boldsymbol{E}=\boldsymbol{I}$. The conversion into such a system is trivial in case of a non singular $\boldsymbol{E}$. For the system (3) an input signal $\boldsymbol{u}(t)$ can be given, so that each arbitrary state $\boldsymbol{q}(T)$ can be reached within the time T starting at $\boldsymbol{q}_{0}=0$. This input signal depends on the so called Gramian controllability matrix $\boldsymbol{W}_{C}(T)$ and can be given as

$$
\boldsymbol{u}(t)=\boldsymbol{B}^{T} \mathrm{e}^{-\boldsymbol{A}^{T} t}\left[\boldsymbol{W}_{C}(T)\right]^{-1} \mathrm{e}^{-\boldsymbol{A} T} \boldsymbol{q}(T)
$$

with

$$
\boldsymbol{W}_{C}(T)=\int_{0}^{T} \mathrm{e}^{-\boldsymbol{A} t} \boldsymbol{B} \boldsymbol{B}^{T} \mathrm{e}^{-\boldsymbol{A}^{T} t} \mathrm{~d} t
$$

The important point for the issue under consideration is that the inputs are somehow connected with the states via the Gramian controllability matrix. Further one the $\mathrm{L}_{2}$-norm for $\boldsymbol{u}(t)$ is

$$
\|\boldsymbol{u}(t)\|_{2}^{2}=\boldsymbol{q}(T)^{T}\left[\boldsymbol{W}_{C}(T)\right]^{-1} \boldsymbol{q}(T)
$$

The Gramian observability matrix $\boldsymbol{W}_{O}(T)$ can be understood in a similar way. It can be used to reconstruct the initial condition $\boldsymbol{q}_{0}$ based on an arbitrary output $\boldsymbol{y}(t)$. This can be given as $(\boldsymbol{u}(t)=0)$

$$
\boldsymbol{q}_{0}=\left[\boldsymbol{W}_{O}(T)\right]^{-1} \int_{0}^{T} \mathrm{e}^{\boldsymbol{A}^{T} t} \boldsymbol{C}^{T} \boldsymbol{y}(t) \mathrm{d} t
$$

with

$$
\boldsymbol{W}_{O}(T)=\int_{0}^{T} \mathrm{e}^{A^{\mathrm{T}} t} \boldsymbol{C}^{T} \boldsymbol{C} \mathrm{e}^{A t} \mathrm{~d} t
$$

and the $\mathrm{L}_{2}$-norm of $\boldsymbol{y}(t)$ is

$$
\|\boldsymbol{y}(t)\|_{2}^{2}=\boldsymbol{q}_{0}^{T} \boldsymbol{W}_{O}(T) \boldsymbol{q}_{0}
$$

Note, that full controllability and full observability have been assumed for the latter considerations. The particular Gramians $\boldsymbol{W}_{C}(\infty)$ und $\boldsymbol{W}_{O}(\infty)$ fulfill the Lyapunov equation

$$
\begin{gathered}
\boldsymbol{A} \boldsymbol{W}_{C}+\boldsymbol{W}_{C} \boldsymbol{A}^{T}+\boldsymbol{B} \boldsymbol{B}^{T}=\mathbf{0} \\
\boldsymbol{A}^{T} \boldsymbol{W}_{O}+\boldsymbol{W}_{O} \boldsymbol{A}+\boldsymbol{C}^{T} \boldsymbol{C}=\mathbf{0}
\end{gathered}
$$

In case of $\boldsymbol{C}^{T}=\boldsymbol{B}$ and a symmetric system of the form (4), the matrixes $\boldsymbol{W}_{C}(\infty)$ und $\boldsymbol{W}_{O}(\infty)$ are identical and can be computed by the generalized Lyapunov equation 


$$
\boldsymbol{E}^{*} \boldsymbol{W}_{C}\left[\boldsymbol{A}^{*}\right]^{T}+\boldsymbol{A} \boldsymbol{W}_{C}\left[\boldsymbol{E}^{*}\right]^{T}+\boldsymbol{B} \boldsymbol{B}^{T}=\mathbf{0}
$$

see [15]. At this point it is important to note, that system (4) und its Gramians are not unique. With each non singular $(2 n \times 2 n)$ matrix $\boldsymbol{T}^{*}$ a transformation in the form of

$$
\boldsymbol{q}=\boldsymbol{T}^{*} \tilde{\boldsymbol{q}}
$$

can be performed which leads to an equivalent but different system. A system is called "balanced" when the Gramians are diagonal and $\boldsymbol{W}_{C}=\boldsymbol{W}_{O}$. The corresponding transformation is unique and the transformation matrix will be denoted as $\boldsymbol{T}$. For the balanced system, Equation (28) evaluates to

$$
\begin{aligned}
\|\boldsymbol{u}(t)\|_{2}^{2} & =\tilde{\boldsymbol{q}}(T)^{T}\left[\begin{array}{ccc}
\frac{1}{\sigma_{1}} & & \\
& \ddots & \\
& & \frac{1}{\sigma_{2 n}}
\end{array}\right] \tilde{\boldsymbol{q}}(T) \\
& =\sum_{i=1}^{2 n} \tilde{q}_{i}(T) \frac{1}{\sigma_{i}} \tilde{q}_{i}(T)
\end{aligned}
$$

with $\sigma_{1}>\sigma_{2}>\cdots>\sigma_{2 n}$. Equation (35) gives insight how the energy of the input signal is distributed among the states which correspond to the vectors of the transformation matrix $T$. A small $\sigma_{\mathrm{j}}$ leads to a large $\sigma_{j}^{-1}$ and that means that the vector $j$ needs a lot of energy from the input signal to be controlled. Equation (31) can be written as

$$
\begin{aligned}
\|\boldsymbol{y}(t)\|_{2}^{2} & =\tilde{\boldsymbol{q}}_{0}^{T}\left[\begin{array}{ccc}
\sigma_{1} & & \\
& \ddots & \\
& & \sigma_{2 n}
\end{array}\right] \tilde{\boldsymbol{q}}_{0} \\
& =\sum_{i=1}^{2 n} \tilde{q}_{0, i} \sigma_{i} \tilde{q}_{0, i}
\end{aligned}
$$

and it gives insight how the energy of the output signal depends from the. A large value of $\sigma_{j}$ means, that the energy of the output signal is strongly influenced by this particular state.

The idea of BT is, to use just these states which need low energy to be controlled from the input and which give a lot of energy to the output. Note, that the values of $\sigma_{j}$ decrease very quickly for common mechanical structures so that just a view columns of the transformation matrix need to be considered as trial vectors for model reduction. A special feature of this approach is an "a priori" error bound in the form of

$$
\|\boldsymbol{y}-\tilde{\boldsymbol{y}}\|_{2} \leq \delta_{B T}\|u\|_{2}
$$

with

$$
\delta_{B T}=2 \sum_{j=m+1}^{2 n} \sigma_{j}
$$

and $m$ as the number of the last considered trial vector, see exemplarily $[10,20,21]$.

Note, that the upper error bound (38) is just valid for a balanced realization of a first order LTI system in the form of (4) or (3) and does not hold when BT is direct applied to second order systems (1). A direct application of BT on second order systems is somehow different. Instead of single controllability and observability Gramian matrixes there are Gramians for the positions and velocities. The interested reader is referred to $[6,13]$ for a detailed discussion and more literature quotations. In $[9,16]$ a modified error bound has been developed for such systems in case of frequency weighted Gramians. For more literature on actual implementations of BT for first order LTI systems and second order systems see [9,10,12-15].

In [35] the use of MM together with BT has been suggested. MM is used there in order to transform a huge system into one of moderate size. In a subsequent step BT has been applied in order to transform the moderate system into one of small size.

The Gramians obtained by the Lyaponov equations consider all frequencies but a direct solution can be very expensive for large systems. It is possible to compute or approximate the Gramians for a frequency range of interest, see [9] or [20]. A low rank approximation of the solution of the Lyaponov equation can be found in [36].

One approach for the reduction of second order systems with an approximated position controllability Gramian is reported in [9] and given next. It delivers valuable qualitative insight into the nature of BT, especially in the framework of un- or lightly damped structures. Similar considerations can be found in [20] for arbitrary first order LTI systems.

In a first step a series of frequency response computations of (1) for $k$ frequencies in the form of

$$
\left(-\omega_{i}^{2} \boldsymbol{M}+\boldsymbol{K}\right) \boldsymbol{X}_{i}=\boldsymbol{B}_{1} \quad 0<\omega_{i}<\omega_{\text {Limit }} 1 \leq i \leq k
$$

have to be carried out. The resulting $(n \times u)$ deformation matrixes $\boldsymbol{X}_{i}$ are collected in the $(n \times(k \times u))$ matrix

$$
\boldsymbol{X}=\left[\begin{array}{llll}
\boldsymbol{X}_{i} & \boldsymbol{X}_{2} & \cdots & \boldsymbol{X}_{k}
\end{array}\right] .
$$

In a subsequent step the matrix $\boldsymbol{X}$ is decomposed in its Proper Orthogonal Modes (POM) by using Proper Orthogonal Decomposition (POD) which is also known as Karhunen-Leove decomposition. The interested readers are referred to [37,38] for a short review on the POD itself and on the available literature. In [39] a detailed outline of the theoretical background can be found. Due to the definition of POD the first $m$ POM $v_{1} \cdots v_{m}$ can be characterized as 


$$
\begin{aligned}
\max _{\boldsymbol{u}_{1}, \cdots, \boldsymbol{u}_{m} \in \mathbb{R}^{n}} & {\left[\left(\boldsymbol{x}_{1}^{T} \boldsymbol{v}_{1}\right)^{2}+\cdots+\left(\boldsymbol{x}_{(k x u)}^{T} \boldsymbol{v}_{1}\right)^{2}+\cdots\right.} \\
& \left.+\left(\boldsymbol{x}_{1}^{T} \boldsymbol{v}_{m}\right)^{2}+\cdots+\left(\boldsymbol{x}_{(k x u)}^{T} \boldsymbol{v}_{m}\right)^{2}\right] \\
\text { s.t. } \quad \boldsymbol{v}_{j}^{T} \boldsymbol{v}_{i}=\delta_{i j} &
\end{aligned}
$$

where $\delta_{i j}$ denotes Kroneckers delta. The vectors $\boldsymbol{v}_{1} \cdots \boldsymbol{v}_{m}$ can be interpreted as those which span "most" of the space of $\boldsymbol{X}$ in a Euclidean sense. Note that the POM are the eigenvectors of $\boldsymbol{X} \boldsymbol{X}^{T}$ and the magnitude of the according eigenvalues correlate with the importance of a POM, analog the entries $\sigma_{i}$ of (35) and (36). For a lot of mechanical systems these eigenvalues decrease rapidly so that $m<<n$. Finally the vectors $\boldsymbol{v}_{1} \cdots \boldsymbol{v}_{m}$ will be used for the reduction matrix $\boldsymbol{V}$. As observed in [9] the matrix $\boldsymbol{X} \boldsymbol{X}^{T}$ is an approximation of the position controllability Gramian which is the link of this approach to BT.

In [40] it has been demonstrated, that the POM of an undamped system with a mass matrix proportional to the identity matrix are identical with the free vibration modes. This observation can be generalized to systems where $\boldsymbol{M}$ and $\boldsymbol{K}$ fulfill the criteria given in [41]. The latter publication focuses on necessary and sufficient conditions so that the vibration modes are orthogonal with respect to the mass and stiffness matrix and to themselves. Note, that the vibration modes orthogonality with respect to themselves is not true in general.

For the following considerations it is assumed that the number of frequency samples $k$ off (39) is high enough so that each reachable vibration mode will be excited nearby its eigenfrequency. Therefore each excited eigenfrequency will lead to huge contributions in $\boldsymbol{X}$. Now it can be concluded that the space spanned by $\boldsymbol{X}$ is dominated by these (linear independed) vibration modes. The later considerations and (41) require that the first and most important POM approximate the space spanned by the dominating vibration modes.

The fact that the conservative system may have infinite response, implies that the "a priori" error estimation (37) is just valid in presence of a viscous damping which is not realistic in the framework of metallic structures, see the remarks in chapter 2.1. This is the first observation why the "error controlled model reduction" is more a theoretical statement than a practical one, when applied to the reduction of metallic structures.

The static response of the structure is undetermined because in general it cannot be ensured that it can be approximated by a space spanned by the vibration modes. Especially when the static response in a dynamical stiff direction is needed, the displacement error may be small but the error in terms of stress is not predictable which is demonstrated in the numerical example below. Note that in a dynamic response computation the exact representation of a static portion is very important because it leads to mean stress which is a significant parameter for fatigue lifetime prediction.

In terms of dynamics, it is known that static deflection shapes, which help to fulfill arbitrary boundary conditions, do accelerate the convergence of a mode base [24]. Therefore it is to expect, that a balanced reduced system does not deliver accurate results in case of mounted or mistuned I/O DOF. The observation that the absence of static displacement fields may lead to considerable errors can be found in [42] as well. This publication deals with the application of POD to linear dynamic systems. The relation to BT is not mentioned there. However, a suggestion is given to improve the reduction base by a static correction.

Finally it is important to emphasize the difference between the eigenfrequencies of (10) and the singular values (SV) $\sigma_{i}$ of (35) and (36). An eigenfrequency has a physical meaning and denotes a pole in the system characterized by $\boldsymbol{M}$ and $\boldsymbol{K}$. The SV describes the importance of a trial vector in terms of a Euclidean distance or an energy transfer from the input to the output. This difference leads to two interesting facts:

- A certain mode will not be detected by BT when the locations of the input/output DOF are at this modes vibration node.

- The sequence of the POM is not directly connected with the eigenfrequencies of the structure but with the location of the input/output DOF. That means that there is no direct correlation between the number of trial vectors and spanned frequency range.

\subsection{Short Remark on the Number of Input/ Output DOF}

As it can be seen in the theory outlined before, the number of I/O DOF is kind of a bottle neck. Too many of such DOF would lead to many columns in the reduction matrixes and therefore to an undesired size of the reduced model. Model reduction for systems with many input/output DOF is still an active field of research and the interested reader is referred to [43-45] for some different approaches to overcome that problem.

\subsection{Summary and Conclusions Based on the Nature of the Three Approaches}

1) Using CMS in a proper way leads to a reduced system which is exact in terms of static and sufficient for a defined range for frequency. It is to expect that the static mode shapes will guarantee satisfying results even if the $\mathrm{I} / \mathrm{O}$ DOF is different mounted as at the time of mode generation.

2) MM leads to an exact static behavior whereas the dynamics is not clearly predictable because no frequency range of validity can be given for a certain number of 
trial vectors.

3) It is to expect, that the strength of MM and BT is the representation of the $\mathrm{I} / \mathrm{O}$ characteristic of the full system in the reduced space. The error is a minimum and even predictable in case of BT when no statics is of significance.

4) For BT it can be expected that the static response is in general not accurate. The dynamic response is not accurate for a reduced system with different boundary conditions as they have been at the time of mode generation.

\section{Numerical Example (Cantilever Beam)}

In the latter section some conclusions have been drawn just by considering the characteristics of the reduction methods. A generic beam example will be investigated for a practical demonstration of the former insights.

As mentioned before a non floating structure (invertible stiffness matrix) is considered in order to keep the formalism as simple as possible without losing generality. There is no single conclusion which would not hold in case of a floating structure.

The beam seems a bit artificial, especially the overhanging sub beam. The intention was the presence of a significant part which is not covered by the input/output DOF. This is of practical relevance for the model reduction of large structures like car bodies or aircrafts. In such cases it cannot be garneted in general, that the critical regions which are not known "a priori" are well represented by the I/O.

\subsection{FE Model and Investigated}

Figure 1 contains a visual representation of the FE model of the beam structure. The beam has been modeled using the CBEAM1 elements of the FE code MSC.NASTRAN [46]. The square of the bottom section is of $100 \mathrm{~mm}$ by

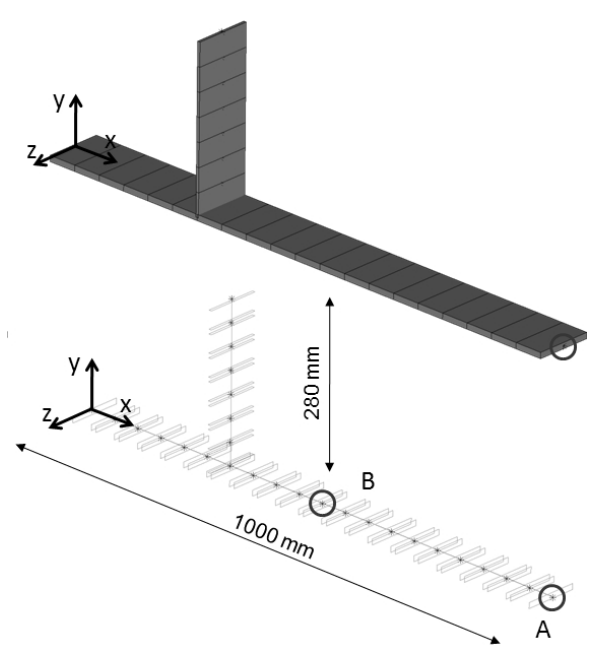

Figure 1. Solid and wireframe representation of the FE model under consideration.
$10 \mathrm{~mm}$ and the one of the overhanging beam is $100 \mathrm{~mm}$ by $5 \mathrm{~mm}$, the Young's modulus is of $210,000 \mathrm{~N} / \mathrm{mm}^{2}$, the shear modulus is of $80,000 \mathrm{~N} / \mathrm{mm}^{2}$ and the density is of $7800 \mathrm{~kg} / \mathrm{m}^{3}$. The structure is mounted at the grid point where the coordinate system is located and 6 input/output DOF are located at the free end of the beam which is denoted as A and marked in Figure 1 by a circle. One additional input/output DOF in $z$ direction is located at the center of the beam which is denoted as B and marked in Figure 1 as well. Note, that the sub beam which has its main extension in $x$ direction will be denoted as "bottom section" further one.

The mass and stiffness matrixes have been assembled by MSC.NASTRAN [46] and exported to an ASCII File. The content of this ASCII File has been imported to Scilab [47] which was used for all subsequent computations.

For the evaluation of the reduction methods, the reduced model will be compared to the full model with respect to statics and dynamics. In order to evaluate the statics, simply the static deflection shapes due to certain loads is computed in the full and in the reduced system. The dynamics is evaluated by a comparison of the eigenvectors and eigenvalues of the full and the reduced system.

Three different variants will be investigated. Note, that the reduction base is equal for all of them. The changes are applied at the reduced model only.

Variant 1: No additional boundary conditions are applied in the reduced system. Consequently the system is equal to the one at the time of mode (or trial vector) generation.

Variant 2: All 6 DOF of point A will be fixed to ground.

Variant 3: All translational and rotational DOF of point A will be fixed to ground except the translation in $x$ direction. This is similar to a "sliding" joint. An additional mass is mounted at point $\mathrm{A}$.

\subsection{Error Measures}

The static results will be compared by plotting the bending lines in the direction of interest. The dynamics of the reduced system will be evaluated by means of the relative error of the frequencies and the MAC value of the normalized mode shapes. The error in frequency of mode number $i$ is measured as

$$
\alpha_{i}=\frac{\tilde{f}_{i}-f_{i}}{f_{i}}
$$

and the MAC value of the corresponding mode shapes is defined as

$$
\beta_{i}=\frac{\left(\varphi_{i}^{T} \tilde{\varphi}_{i}\right)^{2}}{\left(\varphi_{i}^{T} \varphi_{i}\right)\left(\tilde{\varphi}_{i}^{T} \tilde{\varphi}_{i}\right)}
$$


where $\beta_{i}$ has a value between 0 and 1 and 1 means full identity, see [48]. Figure 2 helps to get an impression what $\beta_{i}=0.95$ means in terms of displacements. For that reason, a value of $\beta_{i}<0.95$ will be denoted as inaccurate further one.

\subsection{Model Reduction}

\subsubsection{Moment Matching}

As it can be seen in (21), moment matching leads to blocks which are added to the reduction matrix. Due to the 7 selected I/O DOF the number of trial vectors in the reduction matrix is an even multiple of 7 . For this study a total number of 14 trial vectors have been selected. The trial vectors have been computed along the algorithm cited in [11].

\subsubsection{CMS}

The Craig-Bampton approach [31] requires 7 static displacement vectors and a number of vibration modes which can be estimated by the frequency content of the excitation. As mentioned before, the final size of the entire reduction matrix is given by the MM approach which leads to 7 fixed interface vibration modes which are added to the CMS reduction base. The highest considered frequency is about $331 \mathrm{~Hz}$. Therefore, as an "a priori" estimation, the eigenvalue and eigenvectors should be accurate up to approx. $200 \mathrm{~Hz}$.

\subsubsection{BT}

The trial vectors based on balanced truncation have been computed along the SBPOR algorithm of [15]. This algorithm requires the presence of viscous damping. Therefore a damping matrix $\boldsymbol{D}=1.0 \mathrm{e}^{-4} \boldsymbol{K}$ has been defined in order to obtain the trial vectors.

In Chapter 3.3, it has been assumed, that in case of full controllability the space spanned by balanced truncation is somehow similar to one spanned by the systems vibration modes. For a numerical confirmation of that assumption the first three vibration modes of the structure have been put into a matrix. A singular value decomposition of this matrix delivers the Hankel singular values (HSV) 2.6, 2.2 and 1.4. In a next step the first three trial vectors obtained by the SBPOR algorithm have been added to the matrix which contains the three vibration modes. The HSV of this matrix are 2.7, 2.4, 1.7, 0.006, 0.0001 and 0.0000002 . The sudden decrease of HSV reveals that this 6 dimensional space is dominated by three dimensions. Consequently, both groups of modes

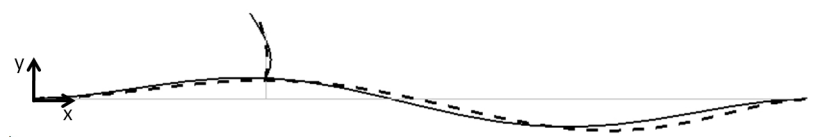

Figure 2. Two mode shapes with $\beta_{i}=\mathbf{0 . 9 5}$. (or trial vectors) span a similar space.

\subsection{Variant 1: Reduced System with the Same Boundary Conditions as at the Time of Mode Generation}

\subsubsection{Static Response}

Two different static load cases will be investigated.

Load Case 1 (LC 1): Two forces in $z$ direction have been applied. One about $50 \mathrm{~N}$ at point $\mathrm{B}$ and another one about $-15 \mathrm{~N}$ at point $\mathrm{A}$.

Load Case 2 (LC 2): A single force in $x$ direction about $1000 \mathrm{~N}$ has been applied at point $\mathrm{A}$.

The resulting deflection in $z$ direction of the bottom section due to LC 1 can be seen in Figure 3. As it was expected the CMS and MM reduced model deliver accurate results while $\mathrm{BT}$ does not.

The resulting deflection in $x$ direction of the bottom section due to LC 2 can be seen in Figure 4. This is a very interesting result. It underlines the advantage of static displacement shapes as trial vectors. While the CMS and MM results are exact the BT result is highly inaccurate. This is, because no trial vector with a dominate displacement in $x$ direction is part of the reduction base. The reason for this is the displacement oriented reduction procedure where directions with high dynamic stiffness are considered to be less important, see (41). The latter observation has important consequences for the dynamoics as well. If an arbitrary dynamic response contains a quasi static portion of the latter deflection in $x$ direction, the resulting stress state will be inaccurate even if the displacement error may be small. The missing mean stress would lead to bad fatigue lifetime prediction as well.

\subsubsection{Dynamics}

Figure 5 contains a summary of the eigenfrequency errors and the correlations of the according mode shapes, respectively. It can be seen that up to $200 \mathrm{~Hz}$, which is the "a-priori" assumed range of validity for CMS, all meth-

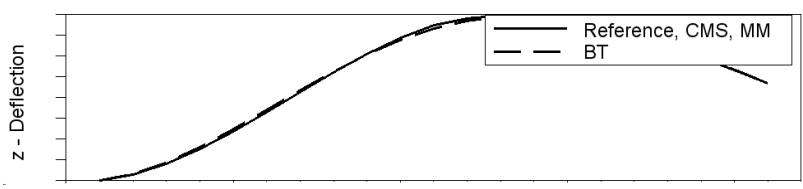

Figure 3. $z$-deflection of bottom section due to LC 1.

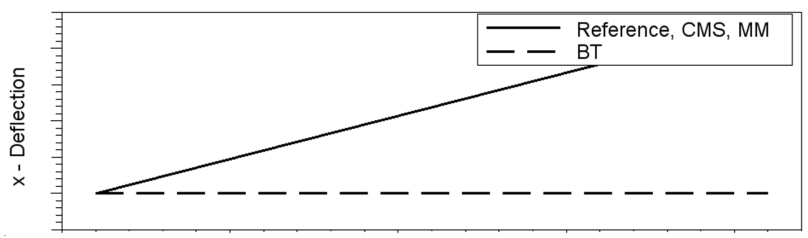

Figure 4. $x$-deflection of bottom section due to LC 2 . 


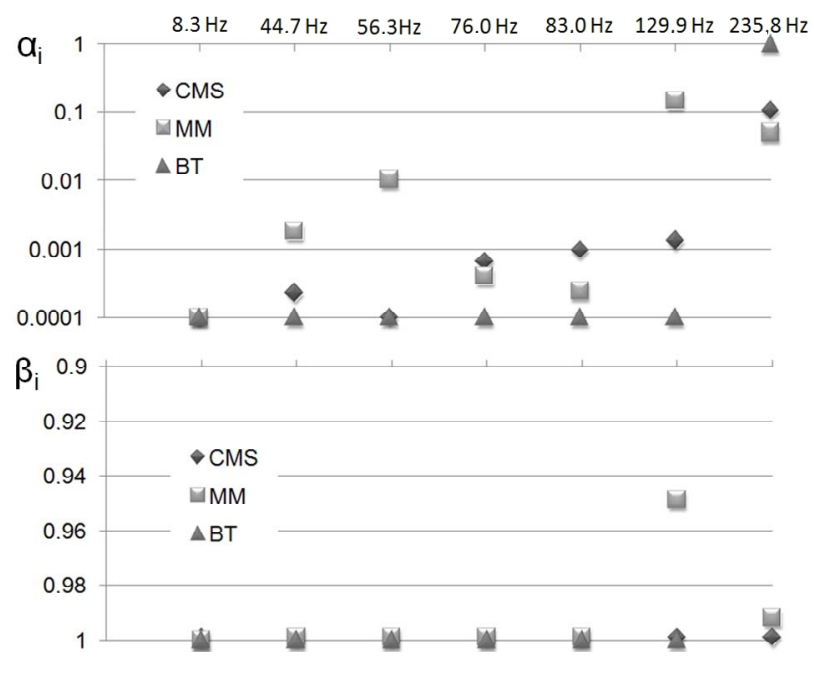

Figure 5. Error in mode frequencies and shapes.

ods deliver acceptable results except MM which leads to a considerable error for the fifth mode at $129.9 \mathrm{~Hz}$. BT gives the most accurate results.

The frequency of mode number 7 is beyond the frequency limit of $200 \mathrm{~Hz}$. However, this mode is not captured by the BT reduction base at all. Both alternative methods provide that mode in the reduced model even the frequency error is considerable. Figure 6 contains a visualization of mode number 7 which is dominated by torsion of the bottom section. It can be observed in the trial vectors $\varphi_{i}$ that the magnitudes of the rotations are much smaller as the ones of the translations. This is related to the chosen units and very common in conventional FE models. The dominating deformations in terms of magnitude are in the overhanging part which is not represented in the input/output which are marked by two circles in Figure 6. This observation leads to the assumption that BT is somehow unity sensitive. This can be easily observed when the relation of BT and POD is considered, see Equation (41). There it can be seen that the importance of trial vectors are based on a Euclidian distance. Therefore the units do play an important role in case of trial vectors which are dominated by rotational part of the I/O DOF only. The absence of this mode will have significant influence on the results of variant 2. Another explanation could be, that the units of the rotations are not in the same scale as the translations.

\subsection{Variant 2: Fixed End}

\subsubsection{Static Response}

One static load case is considered where a single force with $10 \mathrm{~N}$ is acting in $z$ direction of point B. Figure 7 contains the deflection in $z$-direction of the bottom section of the beam. It can be observed, that BT leads to significant errors while CMS and MM deliver accurate results.

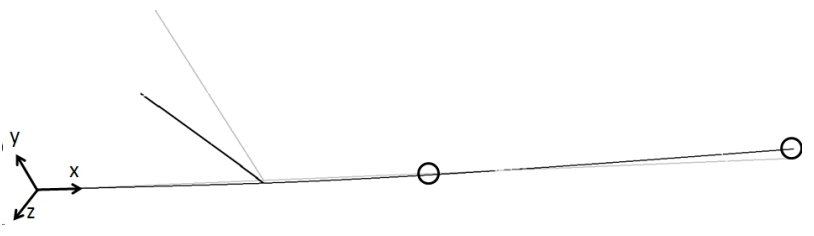

Figure 6. Mode number 7 (torsion oft the bottom section).

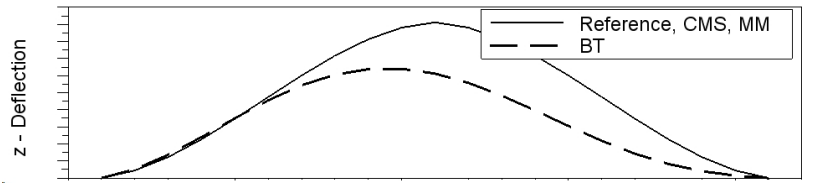

Figure 7. $z$-deflection of bottom section due to a static load.

\subsubsection{Dynamics}

Figure 8 contains a summary of the eigenfrequency errors and the correlations of the according mode shapes, respectively. It can be seen that up to $200 \mathrm{~Hz}$ CMS gives excellent because the investigated modes are part of the reduction base, see (10). MM does not deliver that good performance and the frequency error for the $4^{\text {th }}$ mode is remarkable even it is clear beneath $200 \mathrm{~Hz}$. BT delivers the worst result. This is because the mounted system is badly represented in the mode base without static displacement shapes. Furthermore it can be seen, that mode number 3 is not part of the mode base at all. This is the first torsion mode and the reasons for its absence can be found in the previous subsection. Note, that the missing mode could be excited by an imposed rotational motion at point $\mathrm{A}$. The absence of a mode is always a critical issue if the full system response is of interest.

\subsection{Variant 3: Large Mass Coupling}

In this configuration just 5 DOF of point A will be fixed to ground. The DOF which is associated with the translation in $x$ direction is free and a large point mass about $250 \mathrm{~kg}$ is mounted at point A. The error in mode shapes and frequencies can be seen in Figure 9.

Due to the large mass an additional mode comes into the spectrum of interest. This mode has an eigenfrequency of $145.1 \mathrm{~Hz}$ and represents an elongation of the bottom section along the $x$ direction together with a bending of the overhanging beam, see Figure 10.

It can be observed, that the torsion mode at $98.38 \mathrm{~Hz}$ and the latter mentioned mode at $145.1 \mathrm{~Hz}$ cannot be represented by the BT trial vectors.

Note, that this is not a pure artificial construction. An example would be a flexible slider crank mechanism in a multi body dynamic system (MBDS). The FE model of the elastic slider crank (or con rod) is typically modeled without the mass which will be attached in the MBDS by means of constraints. This mass attachment will influence the modes of the slider crank. 

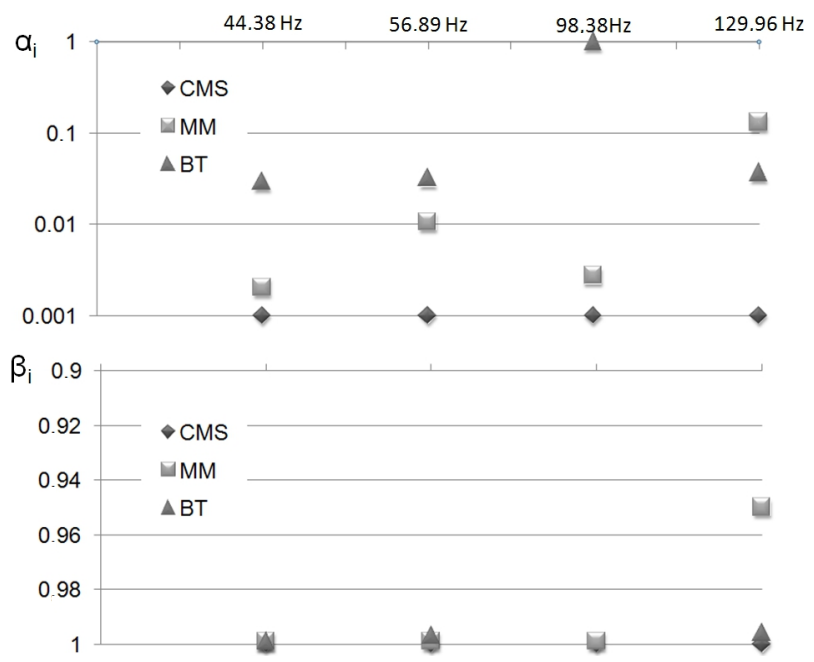

Figure 8. Error in mode frequencies and shapes.
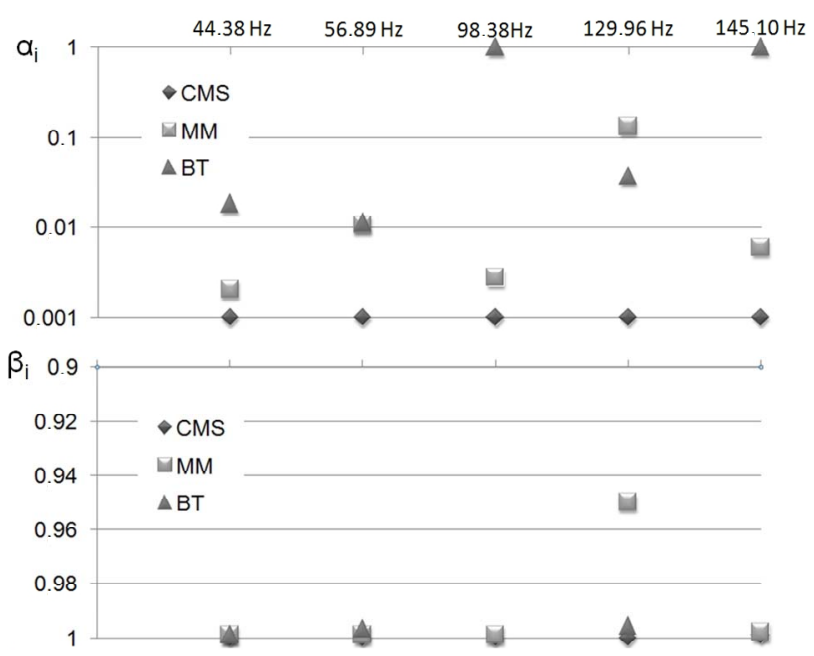

Figure 9. Error in mode frequencies and shapes.

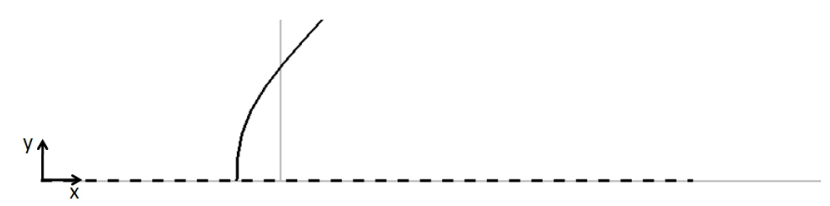

Figure 10. Mode at $145 \mathrm{~Hz}$ (grey represents the reference configuration).

\section{Comments on Former Publications}

In some recent publications MM and BT have been suggested as the somehow better reduction methods in general and for the use in multi body system dynamics in particular. This is in contrast to some of the observations in this work. This will be commented in this section.

\subsection{Frequency Response as Relevant Criterion}

In [16-19] the error in the frequency response functions has been evaluated. This has been done by the compari- son of the reduced models frequency responses with the ones of the full system. All comparisons have in common that the error of BT and MM are much smaller as in case of mode based methods.

Firstly, the evaluation of the frequency response functions do not properly take into account the full system response and boundary conditions which are different as they have been at the time of trial vector generation. The observation in the latter publication matches Figure 5 which holds the eigenfrequencies and mode shape errors of the reduced system. BT and partially MM deliver the best results because the reduced system is not mistuned at the $\mathrm{I} / \mathrm{O}$. As depicted in the sections before a deduction off similar accuracy in case of different boundary conditions is not possible. Further one it could be demonstrated, that the quality of static response is not predictable.

In [18] the mode based methods are represented by the fixed boundary CMS as in this work. The error of this approach is less than $1 \%$ for the frequency range of validity (up to $720 \mathrm{~Hz}$ ). MM and BT deliver better results indeed but the result quality of CMS seems to be sufficient for standard mechanical applications.

The mode based methods in $[16,17,19]$ are not identical with a CMS reduction base in the form of (8). Therefore the error, which goes up to $10 \%$ in the frequency range of interest, cannot be commented here.

\subsection{Time Domain Response as Relevant Criterion}

In [18] a crankshaft is reduced by MM, BT and fixed boundary CMS. A multi body simulation of the full engine fired by gas pressure has been performed. The relative error of the gap and the pressure for one particular lubrication bearing has been evaluated with respect to the time. A close look to the diagrams reveals, that the CMS reduced model has the best performance in an averaged sense. This has to be expected because an operating crankshaft is closer to a mounted crankshaft as to an unsupported one. The conclusion in the latter publication has not been based on this observation but on the frequency response error which is problematic as explained in the subsection before.

\section{Conclusions}

This paper is devoted to a clear answer which method has to be used for the model reduction of metallic structures in context of industrial use. The following conclusions have been drawn by a close look to the underlying theory and they have been demonstrated by means of a generic beam example.

The clear result of the qualitative and quantitative investigations is that modal reduction techniques based on 
component mode synthesis (CMS) have the best overall performance. The results are sufficient accurate in terms of statics and dynamics, independently of the applied boundary conditions. Another practical feature is a clear selection criterion for the number of considered modes as a function of the excitations frequency content.

The observed accuracy in case of moment matching (MM) cannot compete with one obtained by the CMS method. The absence of a correlation between the considered modes and the covered frequency range is another drawback for industrial use where no convergence analysis with respect of the number of trial vectors will be done.

Finally it can be reported, that BT in principle cannot be recommended for model reduction in the investigated framework at all. The reasons are manifold:

If the overall system response contains a static portion, the accuracy in terms of displacement and stress is questionable.

Due to the absence of static correction vectors in the mode base, BT delivers bad results when the I/O DOF in the reduced system face different boundary conditions as at the time of trial vector generation.

Vibration modes can be missed as a consequence of the I/O focused approach and its unity sensitivity. This can lead to unpredictable full system response in case of varying boundary conditions in the reduced system.

There is no correlation between the trial vectors and the covered frequency range.

As a final conclusion it can clearly be stated that the most reliable reduction method for metallic structures and for a wide range of industrial application in mechanical engineering is still the Component Mode Synthesis and its variants. Balanced truncation can be recommended, when the reduced system is not mistuned, when the influence of statics is clarified and when just the $\mathrm{I} / \mathrm{O}$ behavior is of interest.

One of the most important fields where model reduction of flexible bodies needs to be applied is elastic multi body system simulation (MBSS). As already mentioned MBSS is characterized by the importance of statics, overall system response and varying boundary conditions at the I/O DOF of flexible bodies. For the reasons shown in this paper, CMS seems to be the best choice.

The feature of predictable error in case of balanced truncation (BT) may be valuable when the displacement $\mathrm{I} / \mathrm{O}$ behavior of the non reduced system has to be approximated. The practical significance of such an a-priori error estimation is questionable when viscous damping is used as an approximation of real damping, when the boundary conditions in the reduced model differ from the one of the trial vectors and when stresses or statics are of particular interest.

Finally it is mentioned once again, that the focus of the current work is on mechanical engineering. The drawn conclusions may not be valid for other disciplines like control or electrical engineering, where the objectives may be somehow different.

\section{REFERENCES}

[1] A. K. Noor, "Recent Advances and Applications of Reduction Methods," Applied Mechanics Reviews, Vol. 47, No. 5, 1994, pp. 125-146. doi:10.1115/1.3111075

[2] L. Meirovitch, "Computational Methods in Structural Dynamics,” Springer, Berlin, 1980.

[3] Q. Q. Zu, "Model Order Reduction Techniques," Springer Verlag, London, 2004.

[4] R. J. Craig, "Coupling of Substructures for Dynamic Analyses-An Overview," The American Institute of Aeronautics and Astronautics, Vol. 6, No. 7, 2000, pp. 13131319.

[5] R. J. Craig, "A Review of Time-Domain and FrequencyDomain Component Mode Synthesis Methods," International Journal of Analytical and Experimental Modal Analysis, Vol. 2, No. 2, 1987, pp. 59-72.

[6] D. de Clerk, "General Framework for Dynamic Substructuring: History, Review and Classification of Techniques," The American Institute of Aeronautics and Astronautics, Vol. 46, No. 5, 2008, pp. 1169-1181. doi: $10.2514 / 1.33274$

[7] M. Lehner and P. Eberhard, "On the Use of MomentMatching to Build Reduced Order Models in Flexible Multibody Dynamics," Multibody System Dynamics, Vol. 16, No. 2, 2006, pp. 191-211. doi:10.1007/s11044-006-9018-2

[8] R. R. Craig Jr. and T.-J. Su, "Krylov Model Reduction Algorithm for Undamped Structural Dynamics Systems," Journal of Guidance, Control, and Dynamics, Vol. 14, No. 6, 1991, pp. 1311-1313.

[9] M. Lehner, "Modellreduktion in Elastischen Systemen," Doctoral Thesis, Shaker Verlag, Aachen, 2007.

[10] P. Benner, "Numerical Linear Algebra for Model Reduction in Control and Simulation," 2012.

http://www.tu-chemnitz.de/mathematik/preprint/2005/PR EPRINT_18.pdf

[11] B. Lohmann and B. Salimbarhrami, "Ordnungsreduktion Mittels Krylov-Unterraummethoden," Automatisierungstechnik, Vol. 52, No. 1, 2004, pp. 30-38. doi:10.1524/auto.52.1.30.25436

[12] D. G. Meyer and S. Srinivasan, "Balancing and Model Reduction for Second-Order Form Linear Systems," IEEE Transactions on Automatic Control, Vol. 41, No. 11, 1996, pp. 1632-1644. doi:10.1109/9.544000

[13] T. Reis and T. Stykel, "Balanced Truncation Model Reduction of Second-Order Systems," Mathematical and Computer Modelling of Dynamical Systems, Vol. 14, No. 5, 2008, pp. 391-406. doi:10.1080/13873950701844170

[14] P. Benner and J. Saak, "Efficient Balancing Based MOR for Large Scale Second Order Systems, Mathematical and Computer," Modeling of Dynamical Systems, Vol. 17, No. 
2, 2011, pp. 123-143. doi:10.1080/13873954.2010.540822

[15] B. Yan, S. X. D. Tan and B. McGaugy, "Second-Order Balanced Truncation for Passive-Order Reduction of RLCK Circuits," IEEE Transactions on Circuits and Systems-II, Vol. 55, No. 9, 2008, pp. 942-946.

[16] J. Fehr and P. Eberhard, "Error-Controlled Model Reduction in Flexible Multibody Dynamics," Journal of Computational and Nonlinear Dynamics, Vol. 5, No. 3, 2010, p. 31005 .

[17] J. Fehr and P. Eberhard, "Simulation Process of Flexible Multibody Systems with Non-Modal Model Order Reduction Techniques," Multibody System Dynamics, Vol. 25, No. 3, 2011, pp. 313-334. doi:10.1007/s11044-010-9238-3

[18] C. Nowakowski, J. Fehr and P. Eberhard, "Model Reduction for a Crankshaft Used in Coupled Simulations of Engines," In: J. C. Samin and P. Fisette, Eds., ECCOMAS Thematic Conference on Multibody Dynamics, Brussels, 4-7 July 2011, Article ID: 2374-1604.

[19] T. Bonin, M. Zäh, A. Soppa, H. Fassbender, J. Saak and P. Benner, "Modale versus Moderne Ordnungsreduktionsverfahren," Carl Hanser Verlag, München, 2009.

[20] K. Willox and J. Peraire, "Balanced Model Reduction via the Proper Orthogonal Decomposition," AIAA Journal, Vol. 40, No. 11, 2002, pp. 2323-2330. doi: $10.2514 / 2.1570$

[21] H. Faßbender and P. Benner, "Numerische Methoden zur Passivitäterhaltenden Modellreduktion," Automatisierungstechnik, Vol. 54, No. 4, 2006, pp. 153-160. doi:10.1524/auto.2006.54.4.153

[22] C. Tobias and P. Eberhard, "Stress Recovery with Krylov-Subspaces in Reduced Elastic Multibody Systems," Multibody System Dynamics, Vol. 25, No. 4, 2011, pp. 377-393. doi:10.1007/s11044-010-9239-2

[23] P. Fischer, W. Witteveen and M. Schabasser, "Integrated MBS-FE-Durability Analysis of Truck Frame Components by Modal Stresses," Proceedings of the 15th European ADAMS Users' Conference, Rome, 2000. http://www.mscsoftware.com/support/library/conf/adams/ euro/2000/Steyr_Durability_Truck_Frame.pdf

[24] R. Schwertassek, S. V. Dombrowski and O. Wallrapp, "Modal Representation of Stress in Flexible Multibody Simulation," Nonlinear Dynamics, Vol. 20, No. 4, 1999, pp. 381-399. doi:10.1023/A:1008322210909

[25] P. Koutsovasilis and M. Beitelschmidt, "Comparision of Model Reduction Techniques for Large Mechanical Systems," Multibody System Dynamics, Vol. 20, No. 2, 2008, pp. 111-128. doi:10.1007/s11044-008-9116-4

[26] W. Witteveen, "Comparision of CMS, Krylov and Balanced Truncation Based Model Reduction from a Mechanical Application Engineer's Perspective," Conference Proceedings of Society for Experimental Mechanics Series, Vol. 27, 2012, pp. 319-331.

[27] L. Gaul and A. Schmidt, "Experimental Determination and Modeling of Material Damping," VDI-Berichte Nr. 2003, 2007, pp. 17-40.

http://www.iam.uni-stuttgart.de/Mitarbeiter/Schmidt/pape rs/vdi-paper.pdf

[28] D. Ewings, "Modal Testing Theory, Practice and Application," 2nd Edition, Research Studies Press, Baldock, 2000.

[29] S. Bogard, A. Schmidth and L. GauL, "Modeling of Damping in Bolted Structures," VDI-Berichte Nr. 2003, 2007, pp. 97-110.

http://www.iam.uni-stuttgart.de/Mitarbeiter/Schmidt/pape rs/vdi-paper_sergey.pdf

[30] L. Gaul and R. Nitsche, "The Role of Friction in Mechanical Joints," Applied Mechanics Reviews, Vol. 54, No. 2, 2001, pp. 93-105. doi:10.1115/1.3097294

[31] R. R. Craig and M. C. C. Bampton, "Coupling of SubStructures for Dynamic Analysis", AIAA Journal, Vol. 6, No. 7, 1968, pp. 1313-1319. doi:10.2514/3.4741

[32] R. J. Guyan, "Reduction of Stiffness and Mass Matrix," AIAA Journal, Vol. 3, No. 2, 1965, p. 380.

[33] D. J. Rixon, "Dual Craig-Bampton with Enrichment to Avoid Spurious Modes," Proceedings of the IMAC-XXVII, Orlando, 9-12 February 2009, Article ID: 170.

[34] B. Hägblad and L. Eriksson, "Model Reduction Methods for Dynamic Analysis of Large Structures," Computers \& Structures, Vol. 47, No. 4-5, 1993, pp. 735-749.

[35] M. Lehner and P. Eberhard, "A Two Step Approach for Modal Reduction in Flexible Multibody Dynamics," Multibody System Dynamics, Vol. 17, No. 2-3, 2007, pp. 157176. doi:10.1007/s11044-007-9039-5

[36] P. Benner and A. Schneider, "Balanced Truncation Model Order Reduction for LTI Systems with Many Inputs or Outputs," Proceedings of the 19th International Symposium on Mathematical Theory of Networks and Systems, Budapest, 5-9 July 2010, pp. 1971-1974.

[37] G. Kerschen, J. C. Golinval, A. F. Vakakis and L. A. Bergman, "The Method of Proper Orthogonal Decomposition for Dynamical Characterization and Order Reduction of Mechanical Systems: An Overview," Nonlinear Dynamics, Vol. 41, No. 1-3, 2005, pp. 147-169. doi:10.1007/s11071-005-2803-2

[38] A. Chatterjee, "An Introduction to the Proper Orthogonal Decomposition," Current Science, Vol. 78, No. 7, 2000, pp. 808-817.

[39] S. Volkwein, "Model Reduction Using Proper Orthogonal Decomposition," 2012.

http://www.uni-graz.at/imawww/volkwein/POD.pdf

[40] B. F. Feeny and R. Kappagantu, "On the Physical Interpretation of Proper Orthogonal Modes in Vibration," Journal of Sound and Vibration, Vol. 211, No. 4, 1998, pp. 607-616. doi:10.1006/jsvi.1997.1386

[41] F. Ma and C. H. Ng, "On the Orthogonality of Natural Modes of Vibrations," Mechanics Research Communications, Vol. 31, No. 3, 2004, pp. 295-299. doi:10.1016/j.mechrescom.2003.03.001

[42] T. Kim, "Frequency-Domain Karhunen-Loeve Method and Its Application to Linear Dynamic Systems," AIAA Journal, Vol. 36, No. 11, 1998, pp. 2117-2123. doi: $10.2514 / 2.315$

[43] D. M. Tran, "Component Mode Synthesis Methods Using 
Interface Modes. Application to Structures with Cyclic Symmetry," Computers and Structures, Vol. 79, No. 2, 2001, pp. 209-222. doi:10.1016/S0045-7949(00)00121-8

[44] E. Balmes, "Use of Generalized Interface Degrees of Freedom in Component Mode Synthesis," Proceedings of IMAC XIV Conference, Milwaukee, 12-15 February 1996, pp. 204-210.

[45] W. Witteveen and H. Irschik, "Efficient Mode-Based Computational Approach for Jointed Structures: Joint In- terface Modes," AIAA Journal, Vol. 47, No. 1, 2009, pp. 252-263. doi: $10.2514 / 1.38436$

[46] MD R3 Nastran, 2008. www.mscsoftware.com

[47] Scilab, Version 5.3.0. www.scilab.org

[48] R. J. Allemang and D. L. Brown, "A Correlation Coefficient for Modal Vector Analysis," Proceedings of the 1st International Modal Analysis Conference (IMAC I), Orlando, 8-10 November 1982, pp. 110-116. 